\section{Bahnbrechende Daten aus der Uroonkologie}

Bahnbrechende Ergebnisse wurden zur Kombination von Abirateron und Prednison (AP) mit einer Androgenentzugsbehandlung (ADT) beim hormonsensitiven Prostatakarzinom präsentiert. In der LATITUDE-Studie wurden AP plus ADT randomisiert doppelblind und placebokontrolliert verglichen mit ADT alleine bei Patienten mit neu diagnostiziertem, metastasiertem Prostatakarzinom und Hochrisikoprofil (Gleason $\geq 8$; mindestens 3 ossäre Metastasen und/oder viszerale Metastasierung). Bereits in der ersten Interimsanalyse nach 30,4 Monaten zeigte sich ein signifikant längeres medianes Gesamtüberleben (OS) für den experimentellen Behandlungsarm (nicht erreicht vs. 34,7 Monate) (Hazard Ratio [HR] für Todesfall 0,62, $95 \%$-Konfidenzintervall [95\%-KI] 0,51-0,76, p < 0,001). Entsprechend der Empfehlung des Ethikkomitees wurde die Studie frühzeitig beendet und der Kontrollgruppe die zusätzliche Einnahme von AP ermöglicht [Fizazi A et al. ASCO. 2017;LBA 3].

In der STAMPEDE-Studie wurde ebenfalls der zusätzliche Nutzen von AP bei Männern evaluiert, die eine ADT bei metastasierter, nodal-positiver oder -negativer hormonsensitiver Erkrankung erhielten (52\%; $20 \% ; 28 \%)$. Nach einer medianen Beobachtungszeit von $40 \mathrm{Mo}-$ naten wurden 184 Todesfälle bei Patien- ten mit der experimentellen Behandlung und 262 in der Kontrollgruppe verzeichnet (HR 0,63, 95\%-KI 0,52-0,76, $\mathrm{p}<0,001$; HR 0,75 für Patienten mit nicht metastasierter und 0,61 mit metastasierter Erkrankung) [James ND et al. ASCO. 2017;LBA5003]. Somit wird neben Docetaxel nun auch Abirateron Einzug in die Erstlinienbehandlung des hormonsensitiven Prostatakarzinoms halten. Aufgrund eines vergleichbaren Nutzens der beiden Therapien sollten Patientenpräferenz, Begleiterkrankungen und Nebenwirkungsprofil Entscheidungsgrundlage für die Therapiewahl sein.

Beim metastasierten Nierenzellkarzinom stand die Evaluation neuer Kombinationsbehandlungen im Vordergrund. Während sich die Therapie mit der Kombination aus Pembrolizumab und Pazopanib v. a. aufgrund hepatischer Toxizitäten als nicht durchführbar erwies [Chowdhury S et al. ASCO. 2017;Abstr 4506], wurden erste vielversprechende Ergebnisse für Atezolizumab/Bevacizumab sowie Avelumab/Axitinib gezeigt [Atkins MB et al. ASCO. 2017;Abstr 4505; Choueiri TK et al. ASCO. 2017;Abstr 4504]. Beide Kombinationen werden nun in Phase-III-Studien untersucht.

Beim fortgeschrittenen Urothelkarzinom bestätigte sich der Überlebensvorteil unter Pembrolizumab im Vergleich zu einer Zweitlinienchemotherapie nach platinhaltiger Vorbehandlung auch nach längerer Nachbeobachtungszeit [Bajorin DF et al. ASCO. 2017;Abstr 4501]. Epacadostat, ein Hemmstoff eines Tryptophan-katabolisierenden Enzyms, das die T-Zell-vermittelte Immunkontrolle unterdrückt, verbesserte zudem in einer Phase-I/II-Studie mutmaßlich die Effektivität von Pembrolizumab bei insgesamt guter Verträglichkeit [Smith DC et al. ASCO. 2017;Abstr 4503]. Eine entsprechende Phase-III-Studie befindet sich in Vorbereitung.

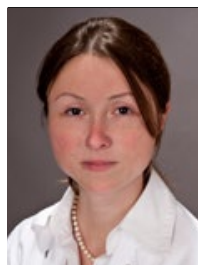

PD Dr. med.

Gunhild von Amsberg

Klinik für Onkologie, Hämatologie und Knochenmarkstransplantation mit Sektion Pneumologie Universitätsklinikum Hamburg-Eppendorf g.von-amsberg@uke.de

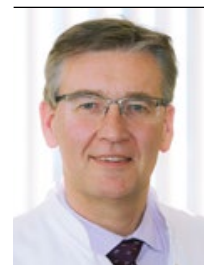

Prof. Dr. med.

Carsten Bokemeyer Klinik für Onkologie, Hämatologie und Stammzelltransplantation mit Sektion Pneumologie, Universitätsklinikum Hamburg-Eppendorf cbokemeyer@uke.de

te-034 [Janjigian YY et al. ASCO. 2017; Abstr 4014] zeigen, dass die PD-1-Inhibitoren Pembrolizumab und Nivolumab aktiv in der Behandlung rezidivierter oder metastasierter Magenkarzinome sind. Die Ansprechraten liegen bei ca. 6-22\%, abhängig vom PD-L1-Expressionsstatus und der Therapielinie. Eine höhere PD-L1-Expression ist assoziiert mit einer höheren Ansprechrate; es können aber auch PD-L1-negative Tumoren auf PD-1-Inhibitoren ansprechen. Mit einer sehr hohen Ansprechrate (ca. 60\%) ist das Vorliegen einer Mikrosatelliteninstabilität assoziiert; aber auch mikrosatellitenstabile Karzinome sprechen an. Die Ergebnisse von Keynote-059 und CheckMate-034 weisen auch auf eine höhere Ansprechrate bei kombinierter 